\title{
Evaluation of Dot Immunoblot for identification of antibodies to Extractable nuclear antigen
}

\author{
Sadia Sharmin1, Sharmeen Ahmed 2, Humayun Sattar 2, Ahmed Abu Saleh 2, Shirin Tarafdar 2 \\ ${ }^{1}$ Department of Microbiology, Ibrahim Medical Collgeg, Shahbag, Dhaka. ${ }^{2}$ Department of Microbiology and Immunology, \\ Bangabandhu Sheikh Mujib Medical University Shahbagh, Dhaka.
}

\begin{abstract}
This cross sectional study was done from January 2009 to June 2010 in Microbiology department of Dhaka Medical The study was designed to evaluate the advantages and reliability of dot immunoblot (DB) for identification of antibodies to extractable nuclear antigens (anti-ENA) in autoimmune connective tissue disorder (CTD). Sera from systemic lupus erythematosus patients $(n=37)$, systemic sclerosis patients $(n=13)$, rheumatoid arthritis patients $(n=11)$, mixed connective tissue disease patients $(n=6)$, polymyositis patients $(n=7)$, and sjogren's syndrome $(n=2)$ were tested for antinuclear antibody (ANA) by Indirect Immunofluorescence (IIF) on HEp-2 cell and analyzed for the presence of antibodies to RNP, Sm, SSA, SSB, Scl-70, and Jo-1 (anti-ENAs) by dot immunoblot and ELISA. Out of 76 sera of autoimmune connective tissue diseases $35(46.05 \%)$ samples were found to be positive by DB and 33 (43.4\%) were positive by ELISA. The dot blot assay exhibited $100 \%$ sensitivity for SSA, SSB, Scl-70 antibodies and for RNP the sensitivity was $90 \%$; specificity was over $98 \%$ for these 4 ENA antibodies. Multiple reactivities were also found in a substantial number of patients sera. No serum was positive for anti-Sm and anti-Jo-1.Healthy controls also revealed no positive reactivity for anti-ENA by DB and ELISA. In conclusion, the dot immunoblot is a valid method for the detection of specific autoantibodies in autoimmune connective tissue disorder. Its most notable advantages are simultaneous detection of several autoantibodies in the same serum and its lower cost.
\end{abstract}

Key Words: Dot Immunoblot, nuclear antigen, ELISA.

\section{Introduction}

Indirect immunofluorescence (IIF) on HEp-2 cell is the classically used technique for the detection of antinuclear antibody (ANA) in autoimmune connective tissue disorder (CTD). Positive fluorescence staining indicates the presence of ANA but does not allow precise identification of specific autoantibodies. For that purpose, additional testing is required, employing techniques such as immunoprecipitation in agar, western immunoblotting, enzyme linked immunosorbent assay (ELISA) or, the more recently developed, dot immunoblot (DB) and line immunoassay ${ }^{1,2,3}$. ANA, which show many different serologic specificities, are partly directed against extractable nuclear antigens (ENA) most commonly SSA, SSB, Sm, RNP, Jo-1 and Scl-70. Because a characteristic profile of ANA is associated with most CTD, identification of the fine specificity may provide

Correspondence:

Dr. Sadia Sharmin

Assistant Professor

Department of Microbiology

Ibrahim Medical College, Shahbag, Dhaka. valuable clues to the diagnosis. Indeed, the presence of antidsDNA or anti-Sm antibodies is quite specifically associated with systemic lupus erythematosus (SLE); anti-Scl70 and anticentromere antibodies with systemic sclerosis (diffuse and limited cutaneous disease respectively; anticentromere antibodies, however, can also be seen in patients without this disease); anti-Jo-1 with polymyositis. Anti-SSA and anti-SSB antibodies have a broader spectrum of associated CTD, including sjogren's syndrome (SS), lupus (systemic and cutaneous form), scleroderma, and rheumatoid arthritis (RA). Anti-RNP antibodies have been associated with the so-called mixed connective tissue disease (MCTD), but may be found in other rheumatic connective tissue diseases as well ${ }^{1}$.

In this study, samples of autoimmune connective tissue disease patients were analyzed for ANA testing and further identification of the fine reactivity of these antibodies were obtained with Dot immunoblot and ELISA (for detection of antibodies to extractable nuclear antigens).

The present study was carried out to assess the usefulness of DB for detection of anti-ENA. The purpose was not to replace ELISA, which has several advantages, but to evaluate the reliability of DB as a diagnostic tool for identification of 
anti-ENA in autoimmune connective tissue disease. The main advantage of the DB technique is that it allows the simultaneous identification of different autoantibodies and each one has not to be studied individually in each serum.

\section{Materials and Methods}

\section{Patients}

A total of 76 patients of autoimmune CTD, attending at lupus clinic in BSMMU during the study period of May, 2010 to October, 2010; were enrolled in this cross sectional study. The study group comprised 37 patients with systemic lupus erythematosus (SLE), diagnosed according to the American College of Rheumatology classification criteria, 39 patients with other systemic rheumatic diseases (other SRD). The group of other SRD consisted of 13 patients with systemic sclerosis (SSc), 11 with rheumatoid arthritis (RA), 07 with polymyositis (PM), 06 with mixed connective tissue diseases (MCTD) and 02 with sjogrens syndrome (SS). All clinical records of the patients including hematological, biochemical and serological investigations were assessed. In addition, sera of 20 healthy controls were included in this study. Blood (5 $\mathrm{ml}$ ) was drawn and sera were separated from the clotted blood samples of all SLE patients by centrifugation. Sera were stored at $4^{\circ} \mathrm{C}$ if testing was planned within 72 hours or at $-20^{\circ} \mathrm{C}$ for testing after three days (without freezing and thawing). Each of the serum samples was subjected for ANA testing by Indirect Immunofluorescence on HEp-2 cell followed by Dot immunoblot and ELISA for anti-ENA.

\section{Indirect immunofluorescence on HEp-2 cell}

Indirect immunofluorescence was done by a commercially available kit. Serum diluted $1 / 40$ in phosphate buffered saline (PBS) was overlaid onto fixed HEp-2 cell (ALPHADIA, Belgium) for 30 minutes at room temperature. Slides were washed twice for five minutes each with PBS, overlaid with fluorescence labeled conjugate, which is antihuman IgG heavy and light chain specific and incubated for an additional 30 minutes. After washing twice, a coverslip was placed over the slide, and the slides were read using a fluorescence microscope at' 40 power ${ }^{4}$. The main fluorescence patterns seen were speckled, homogeneous, peripheral and nucleolar.

\section{Dot Immunoblot}

The Dot immunoblot method is a qualitative assay, which utilizes strips of nitrocellulose on which purified antigens are blotted at prelocated spots. Coated antigens used in this study were Sm, RNP, SSA, SSB, Jo-1 and Scl-70. The antigen sources used are bovine and rabbit thymus (SSA and Sm) or calf spleen and rabbit thymus (SSB and Sm/RNP). The test procedure was performed according to directions supplied by the manufacturer (D-tek, Belgium). Test strips were incubated for 10 minutes with a diluted patient serum in a PBS-Tweeen solution. Subsequently the test strips were washed by gentle agitation in a test tube filled with PBSTween for $1 \mathrm{~min}$. After the excess buffer solution was removed with a filter paper, the test strips were incubated with an alkaline phosphatase-Protien A conjugate for $10 \mathrm{~min}$. The test strips were then washed for $1 \mathrm{~min}$ by gentle agitation by PBS-Tween. Again excess buffer was removed with filter paper. Finally the test strips were stained with 5-bromo-4chloro-3-indolylphosphate for $5 \mathrm{~min}$. The reaction was terminated by washing the test strips with deionized water. The strips were then air dried. Only strips on which the positive control position was stained as a clearly marked blue spot were able to be evaluated and used for this study ${ }^{5}$.

\section{ELISA}

ELISA is a quantitative method for detection of anti-ENA and was detected by commercially available ELISA kit (Orgentec, Germany). Highly purified specific ENA is bound to microwells. Antibodies against this antigen, if present in diluted serum, bind to the respective antigen. Washing of the microwells removes unspecific serum components. Horseradish peroxidase (HRP) conjugated antihuman IgG immunologically detects the bound patient's antibodies forming a conjugate/antibody/antigen complex. Washing of the microwells removes unbound conjugate. An enzyme substrate in the presence of bound conjugate hydrolyses to form a blue colour. The addition of an acid stops the reaction forming a yellow end-product. The intensity of this yellow colour is measured photometrically at $450 \mathrm{~nm}$. The amount of colour is directly proportional to the concentration of antibodies present in the sample.

\section{Statistical analysis}

Sensitivity is defined as the probability of a positive test result in a patient with the disease under investigation. Sensitivity is calculated as the number of test-positive samples within the disease population (true-positives), divided by the total number of samples from patients with the respective disease (true-positives + false-negatives). Specificity is the probability of a negative test result in a patient without the disease under investigation. Specificity is calculated as the number of test-negative samples within the population without the disease (true-negatives), divided by the total number of samples from patients without the disease (false-negatives + true-negatives).

\section{Results}

In this cross sectional study, 37 samples of SLE patients, 13 with systemic sclerosis (SSc), 11 with rheumatoid arthritis (RA), 07 with polymyositis (PM), 06 with mixed connective 
tissue diseases (MCTD) and 02 with sjogrens syndrome(SS) were analyzed. A total of $35(46.05 \%)$ samples were found to be positive by dot blot and $33(43.4 \%)$ were positive by ELISA among 76 sere of CTD patients. The comparison between DB and ELISA for the individual ENA antibodies is presented in table I.

Table-1: Dot Immunoblot Reactivity in Relation to ENA Antibody

\begin{tabular}{lcccc}
\hline \multirow{4}{*}{ Antibody } & $\begin{array}{c}\text { Concordant results } \\
\text { Dot blot positive } \\
\text { ELISA positive }\end{array}$ & $\begin{array}{c}\text { Dot blot negative } \\
\text { ELISA negative }\end{array}$ & $\begin{array}{c}\text { Dot blot positive } \\
\text { ELISA negative }\end{array}$ & $\begin{array}{c}\text { Dot blot positive } \\
\text { ELISA positive }\end{array}$ \\
\hline Sm & 0 & 0 & 0 & 0 \\
RNP & 9 & 66 & 0 & 1 \\
SSA & 4 & 71 & 1 & 0 \\
SSB & 2 & 74 & 0 & 0 \\
Jo-1 & 0 & 0 & 0 & 0 \\
Scl-70 & 6 & 69 & 1 & 0 \\
Multiple & 11 & 64 & 1 & 0 \\
autoantibod ies & & & \\
\hline
\end{tabular}

The sensitivity, specificity, positive predictive value and negative predictive value of the DB were calculated by comparison with the ELISA and are presented in table II.

Table-2: Comparison of Dot immunoblot with the ELISA technique

\begin{tabular}{|c|c|c|c|c|}
\hline & Anti-RNP & Anti-SSA & Anti-SSB & Anti-Scl-70 \\
\hline Sensitivity & $90 \%$ & $100 \%$ & $100 \%$ & $100 \%$ \\
\hline Specificity & $100 \%$ & $98.6 \%$ & $100 \%$ & $98.5 \%$ \\
\hline $\begin{array}{l}\text { Positive } \\
\text { predictive value }\end{array}$ & $100 \%$ & $80 \%$ & $100 \%$ & $85.7 \%$ \\
\hline $\begin{array}{l}\text { Negative } \\
\text { predictive value }\end{array}$ & $98.5 \%$ & $100 \%$ & $100 \%$ & $100 \%$ \\
\hline
\end{tabular}

Overall the sensitivity and specificity of DB for detection of specific ENA antibodies were excellent with the exception of sensitivity for RNP. The dot blot assay exhibited $100 \%$ sensitivity for SSA, SSB, Scl-70 antibodies and 90\% for RNP; specificity was over $98 \%$ for all these 4 ENA antibodies. No serum was positive for anti-Sm and anti-Jo-1 but a substantial number of patient samples were positive for multiple autoantibodies. However, as no gold standard for ANA characterization exists, one has to be careful with the interpretation of these data.

ENAs in Healthy Control Samples

With regard to the specificity of the technique evaluated in this study, we examined reactivity in healthy control samples $(n=20)$. The DB and ELISA did not show any positive reaction for these samples.

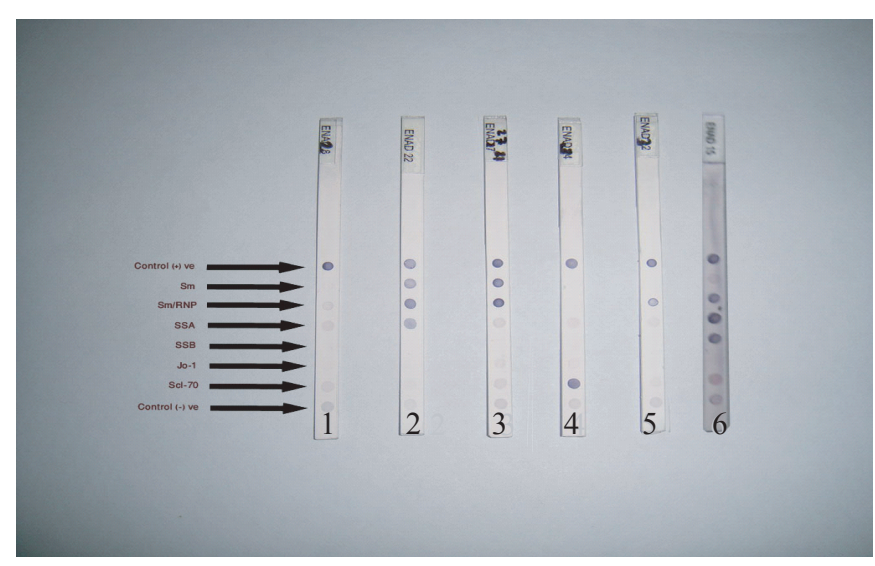

Fig.1-Dot Blot strips. Strip1-negative; Strip 2-Anti-Sm,RNP,SSA positive; Strip 3-Anti-Sm,RNP positive; Strip 4-Anti-Scl-70 positive; Strip 5-Anti-RNP positive; Strip 6-Anti-RNP,SSA,SSB positive

\section{Discussion}

Antibodies against extractable nuclear antigens (ENAs) are useful diagnostic markers for various autoimmune connective tissue diseases. The antibodies can be identified by either counter immunoelectrophoresis (CIE) or immunoblotting. These techniques, however, are timeconsuming, technically demanding, and do not provide for fast turnaround times. Over the last few years, alternative techniques such as ELISA and dot blot (DB) have been introduced ${ }^{6}$. A DB technique has been evaluated for autoantibodies against ENAs by Ermens et al and Mewis et al ${ }^{5,6}$. In this study, we tried to evaluate DB for detection of ENAs in sera of patients with autoimmune connective tissue diseases, that is, SLE, SSc, PM, MCTD, RA, SS and healthy controls. The results reveal that sensitivities for the conventional ENAs, as obtained by DB quite similar to ELISA. Sensitivity and specificity of DB assay found in this study is similar to the result described by Ermens et al and Mewis et al in their study ${ }^{5,6}$. Although antibodies to SSA are not specific for any of the autoimmune connective tissue diseases, these antibodies may be prognostic for the development of neonatal lupus and/or a congenital heart block $^{7,8}$. Interestingly, it has been reported recently that maternal autoantibodies directed to a specific epitope within the leucine zipper amino acid sequence 200-239 (p200) of the SSA protein correlate with prolongation of fetal atrioventricular time and heart block. ${ }^{9}$ This indicates that detection of antibodies specific for the SSA subunit is clinically relevant in pregnant women. As DB can detect different autoantibodies simultaneously in same 
serum, it is advantageous to use DB to detect anti-ENA in pregnancy. The DB test is advantageous for time management as described previously for a similar test system for ENA antibodies ${ }^{10}$. The test procedure could easily be performed in batch assays as every 5 min a new DB test could be started. A major drawback of the DB method is the blotting of RNP antigen in combination with $\mathrm{Sm}$ antigen (Fig.1). This implies that if both the Sm spot and the Sm/RNP spot are positive the presence of Sm antibodies alone cannot be distinguished from the combined presence of Sm and RNP antibodies. 5 The DB assay evaluated in this study has the advantage over the other techniques is that it enables the detection of antibodies with additional specificities. In conclusion, the dot blot assay is a valid method for the simultaneous detection of various autoantibodies in autoimmune connective tissue disorder. Its major advantages with respect to ELISA method are its lower price and the fast and simultaneous detection of different autoantibodies in a single serum. The identification of autoantibodies with DB could contribute to reducing the bias derived from using different methods of detection when series of patients are compared.

\section{References:}

1. Von Mühlen CA, Tan EM. Autoantibodies in the diagnosis of systemic rheumatic diseases. Semin Arthritis Rheum 1995; 24:323-58.

2. Meheus L, Van Venrooij WJ, Wiik A, Charles PJ, Tzioufas AG,Meyer O, et al. Determination of the fine specificity of antinuclear antibodies in connective tissue diseases using a multiparameter line immunoassay (LIA) based on recombinant proteins. Clin Exp Rheumatol 1999; 17:205-14.

3. Tan EM, Smolen JS, Mc Dougal JS, Butcher BT, Conn D, Dawkins $\mathrm{R}$, et al. A critical evaluation of enzyme immunoassays for detection of antinuclear antibodies of defined specificities. Arthritis Rheum 1999; 42:455-64.
4. Peene I, Meheus L, Veys EM, Keyser FDe. Detection and identification of antinuclear antibodies (ANA) in a large and consecutive cohort of serum samples referred for ANA testing. Ann Rheum Dis. 2001; 60:1131-1136.

5. Ermens AAM, Bayens AJM, van Gemert ACM, van Duijnhoven LP. Simple dot-blot method evaluated for detection of antibodies against extractable nuclear antigens. Clin Chem 1997; 12:2420-2.

6. Mewis A, Marien G, Blanckaert N, Bossuyt X. Evaluation of a Dot-Blot Method for Identification of Antibodies against Extractable Nuclear Antigens and AntiCytoplasmic Antibodies. Clinical Chemistry 1999; 45(2).

7. Watson RM, Lane AT, Barnett NK, et al. Neonatal lupus erythematosus: a clinical, serological and immunogenetic study with review of the literature. Medicine 1984; 63: 362-378.

8. Singsen BH, Akhter JE, Weinstein MM, et al. Congenital complete heart block and SSA antibodies: obstetric implications. Am. J. Obstet. Gynecol . 1985 ; 152: $655-658$.

9. Salomonsson S, Sonesson SE, Ottosson L, et al. Ro/SSA autoantibodies directly bind cardiomyocytes, disturb calcium homeostasis, and mediate congenital heart block. J. Exp. Med 2005; 201: 11-17.

10. Paxton H, Bendele T, O'Connor L, Haynes DC. Evaluation of the rheumastrip ANA profile test: a rapid test strip procedure for simultaneously determining antibodies to autoantigens U1-ribonucleoprotein (U1RNP), Sm, SS-A/Ro, SS-B/La, and to native DNA. Clin Chem 1990; 36:792-797. 